\title{
GV/m Single-Cycle Terahertz Fields from a Laser-Driven Large-Size Partitioned Organic Crystal
}

\author{
Carlo Vicario, ${ }^{1}$ Balazs Monoszlai, ${ }^{1}$ and Christoph P. Hauri ${ }^{1,2}$ \\ ${ }^{1}$ Paul Scherrer Institute, SwissFEL, 5232 Villigen PSI, Switzerland \\ ${ }^{2}$ Ecole Polytechnique Federale de Lausanne, 1015 Lausanne, Switzerland
}

(Received 12 February 2014; published 29 May 2014)

\begin{abstract}
We report on compact and efficient laser-based $\mathrm{THz}$ generation in the terahertz frequency gap (1-10 THz). The radiation is generated by optical rectification of a midinfrared laser in a large-size, partitioned nonlinear organic crystal assembly. This enables up-scaling of presently field-limited tabletop $\mathrm{THz}$ sources to $\mathrm{GV} / \mathrm{m}$ electric and several tesla magnetic field at millijoule pulse energy. In agreement with simulations, the $\mathrm{THz}$ beam properties at focus are shown to be not deteriorated by the discontinuity of the emitter surface. The high laser-to-THz energy conversion efficiency exceeds the Manley-Rowe limit and is explained by a cascaded $\chi^{(2)}$ process in the organic crystals accompanied by a significant redshift of the pump spectrum. The scheme provides a compact, tabletop $\mathrm{THz}$ source for single-cycle transients at field strength equivalent or even higher to linear accelerator and FEL-based THz sources. This opens an avenue toward novel nonlinear $\mathrm{THz}$ applications.
\end{abstract}

DOI: 10.1103/PhysRevLett.112.213901

PACS numbers: 42.65.Re, 42.25.Bs, 42.65.Ky, 42.72.Ai

Single-cycle terahertz $(\mathrm{THz})$ pulses at high fields have recently attracted large attention since they offer novel opportunities in photonics, condensed matter physics, in biology and surface chemistry. The advent of hundred $\mathrm{MV} / \mathrm{m}$ high-field pulses in the previously hardly accessible frequency range $(1-10 \mathrm{THz})$ has opened an avenue to excite ultrafast dynamics by phonons and resonant modes [1]. Higher $\mathrm{THz}$ field strengths above the $\mathrm{GV} / \mathrm{m}$ barrier are expected to become a formidable tool for broader applications in physics, including laser-based particle acceleration in dielectric structures, ultrafast nonresonant magnetic domain switching of advanced materials (e.g., multiferroics, ferroelectrics) and the investigation of modern electronic devices where voltages of $\sim 1 \mathrm{~V}$ pernm $(\sim 1 \mathrm{GV} / \mathrm{m})$ are applied during ultrashort time scales.

The most prominent laser-based approaches providing intense $\mathrm{THz}$ transient at a hundred $\mathrm{MV} / \mathrm{m}$ field strength are based on nonlinearities in plasma [2], optical rectification $(\mathrm{OR})$ in lithium niobate (LN) $[3,4]$ and, more recently, OR in organic crystals [5-9]. Since the quest for higher field strength is incessant the THz pulse energy needs to be enlarged. Up-scaling of the $\mathrm{THz}$ output energy calls for increasing the $\mathrm{THz}$ emitting surface and pump spot size while keeping the pump fluence below the damage threshold. Not all of the conventional laser-based terahertz sources offer this opportunity. OR in cryogenically cooled $\mathrm{LN}$, for example, is efficient [10] but the potential for upscaling the $\mathrm{THz}$ output seems restricted as it requires enhanced control on the pulse front tilt for large beams and the production of large defect-free crystals. This is presently a formidable challenge [11,12]. Furthermore the frequency range in $\mathrm{LN}$ is limited to $\leq 1 \mathrm{THz}$ due to significant absorption at higher frequencies, and tight focusing is challenging due to the elliptically shaped intensity profile and unequal transverse beam divergences in $x$ and $y$. While plasma sources are widely tunable they only offer limited $\mathrm{THz}$ pulse energy $(<\mu \mathrm{J})$ which seems difficult to up-scale. On the other hand, the organic $\mathrm{THz}$ emitters (DAST, DSTMS, OH1, HMQ-TMS [5-9,13]) offer a number of benefits. They provide high laser-to$\mathrm{THz}$ conversion efficiencies at room temperature and octave-spanning spectra, they do not require sophisticated pump pulse shaping (i.e., no pulse front tilting and imaging) and the $\mathrm{THz}$ radiation is naturally collimated and aberration-free. This makes it possible to focus the beam by a single optics to diffraction-limited spot-size and to create high field strength. However, up to now the maximum $\mathrm{THz}$ energy was limited by the limited available aperture size of those organic crystals.

In this Letter, we report on a new concept for resolving current limitations in $\mathrm{THz}$ pulse energy and field strength from laser-driven organic crystals and present a complete source characterization. Our approach is based on combining coherently the output of multiple, small DSTMS organic crystals in a large size $\mathrm{THz}$ emitter. Pumped with our powerful mid-infrared laser the presented THz source emits in the hardly accessible THz frequency gap (1-10 THz) and provides record-high $170 \mu \mathrm{J}$ pulse energy per $\mathrm{cm}^{2}$ crystal surface. The resulting field strength reaches $0.6 \mathrm{GV} / \mathrm{m}$ within one single carrier oscillation, limited only by the available laser power. In our experiment the large-size, partitioned $20 \times 20 \mathrm{~mm}^{2}$ OR surface offers a THz emission area 10 times bigger than a conventional DSTMS crystal. Pumping the entire, partitioned crystal surface (PCS) results in $680 \mu \mathrm{J} \mathrm{THz} \mathrm{energy} \mathrm{in} \mathrm{an} \mathrm{extremely} \mathrm{compact} \mathrm{setup} \mathrm{at} \mathrm{field}$ strength of $3.6 \mathrm{GV} / \mathrm{m}(12 \mathrm{~T})$ and $3 \mathrm{TW} / \mathrm{cm}^{2}$ peak intensity 
for a diffraction-limited spot size. We show that the high conversion efficiency is due to a cascaded optical rectification process. In previous work [14] a tiny mosaic-patterned crystal provided sub-kV/m electric field strength. By developing a significantly larger pattern we experimentally demonstrate here an up-scaling of the field strength by more than six orders of magnitudes and show that the individual electric fields sum up coherently in the focal region. Compared to particle accelerator based sources $[15,16]$, the tabletop, laser-based scheme presented here is superior for THz/optical pump-probe experiments due to intrinsic synchronization, the accessibility and repetition rate.

In the experiment, performed at the Paul Scherrer Institute, we use a multiterawatt Ti:sapphire laser front end [17] with a subsequent optical parametric amplifier (OPA), Fig. 1. The collimated multi-mJ beam of the OPA $(3 \mathrm{~mJ}, 65 \mathrm{fs}, 5 \mathrm{~mm}, 100 \mathrm{~Hz}$ ) at $1.5 \mu \mathrm{m}$ wavelength serves as pump for the PCS crystal. The PCS consists of multiple parts of uncoated, small DSTMS crystals. These pieces are glued with a transparent adhesive on the host substrate (fused silica) to cover a total surface of $400 \mathrm{~mm}^{2}$. The substrate is antireflective coated at the pump laser wavelength and is positioned toward the OPA to avoid the terahertz reabsorption in the fused silica. The $5 \mathrm{~mm}$ thick host substrate transmits more than $90 \%$ pump energy and introduces negligible group velocity dispersion at the pump wavelength. The single DSTMS crystals are oriented such that they exhibit common crystallographic axes to provide optimum phase-matching orientation across the entire PCS area. The individual organic crystal pieces are spaced by about $200 \mu \mathrm{m}$ and have a thickness of $500 \pm 15 \mu \mathrm{m}$.

The temporal and spectral shapes of the THz field from the PCS are shown in Fig. 2. Pumped by the full midinfrared energy available from our laser the $\mathrm{THz}$ pulse

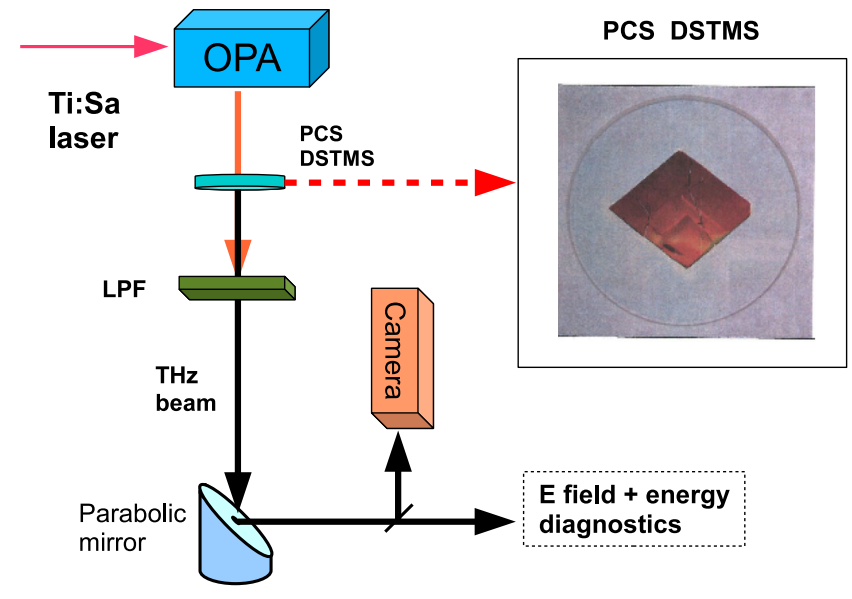

FIG. 1 (color online). Tabletop terahertz source based on the partitioned DSTMS (PCS, shown in the inset). The crystal is pumped by an optical parametric amplifier (OPA). The THz beam is separated from the residual pump by a low-pass filter (LPF) and characterized by an electro-optical sampling, a $\mathrm{THz}$ camera, a Golay cell, and a $\mathrm{THz}$ autocorrelator. reaches field strengths of $0.6 \mathrm{GV} / \mathrm{m}$ and $2 \mathrm{~T}$ in a single carrier oscillation [Fig. 2(a)]. The THz field evolution is reconstructed in dry air using an electro-optical sampling in $100 \mu \mathrm{m}$ thin $\mathrm{GaP}$ crystal and the field strength is calculated from the $\mathrm{THz}$ spot size, the pulse duration and energy. The multioctave spanning $\mathrm{THz}$ spectrum [Fig. 2(b)] originating from the large-size fragmented structure peaks at $3.2 \mathrm{THz}$ and covers frequencies up to $8 \mathrm{THz}$. The dips at around $1 \mathrm{THz}$ and $5 \mathrm{THz}$ are due to absorption in the organic crystal due to phonon active modes [7]. The emission properties of the PCS are compared, at identical pump fluence, to a singlet small-size DSTMS crystal $\left(3 \times 3 \mathrm{~mm}^{2}\right)$ of same thickness. The comparison reveals similar field and spectral shapes in the focus but 3 times larger field strength for the PCS. This gives a first indication that the $\mathrm{THz}$ field characteristics are not spoiled by the discontinuities in the PCS emitter neither by negative interference of fields emitted from different crystal parts.

In principle tight focusing could be hampered by wavefront aberrations caused by discontinuities and by errors in orientations and thickness of the individual PCS crystals. In order to verify the influence of these effects on beam quality we investigated the $\mathrm{THz}$ beam distortions by recording the intensity beam profile in the near field and
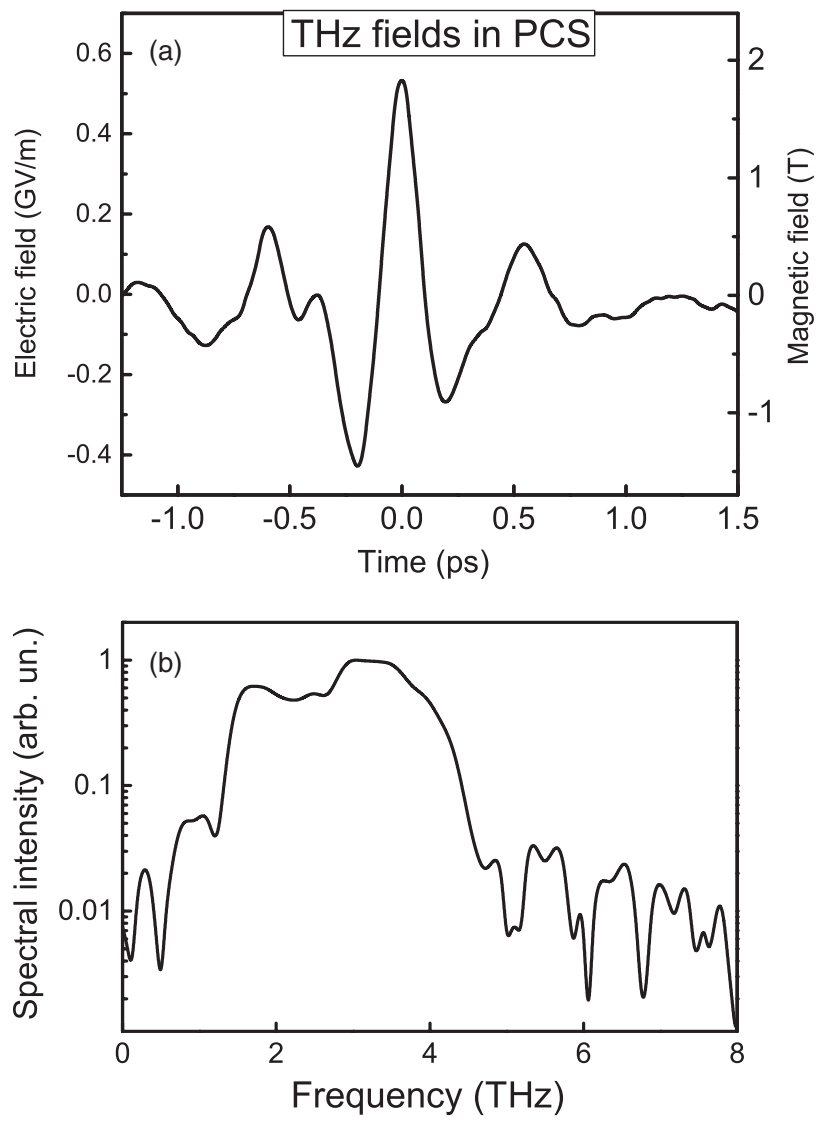

FIG. 2. THz electric field (a) and corresponding spectrum (b) obtained by optical rectification in a patterned DSTMS crystal structure. 

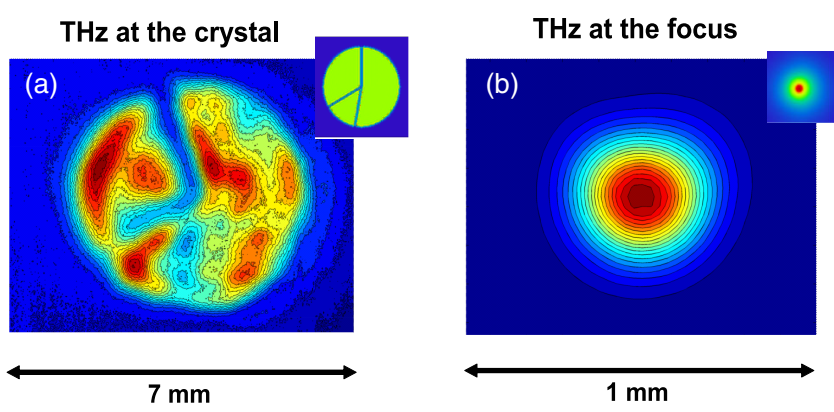

FIG. 3 (color online). Measured $\mathrm{THz}$ distribution at (a) the crystal output and at (b) the focus of parabolic mirror with numerical aperture of 0.5 . The insets show the simulated intensity profile at the same position.

the far field. Shown in Fig. 3(a) is the measured near-field profile, which has been recorded by imaging the PCS crystal surface onto an uncooled microbolometer $\mathrm{THz}$ imager (NEC Corporation, IRV-T0830, $23.5 \mu \mathrm{m}$ pixel pitch). The gaps between three crystals are clearly visible. The additional intensity variation observed across the emitting area originates from disuniformities in the pump beam. The $\mathrm{THz}$ beam generated from three separated crystals in the near-field results in three beams propagating collinearly. These beams are measured to combine perfectly in a single intensity spot at the focus point behind an offaxis parabolic mirror (numerical aperture of 0.5 , focus of $100 \mathrm{~mm}$ ), as shown in Fig. 3(b). The corresponding circular $\mathrm{THz}$ intensity profile at the focus is measured to be $270 \mu \mathrm{m}$ (FWHM), which is close to its diffraction limit. The experimental results are reproduced by modeling done using ZEMAX optical design code [insets in Figs. 3(a) and 3(b)]. To model the $\mathrm{THz}$ source the emitted beam was sliced into discrete frequencies between $0.5-0.6 \mathrm{THz}$ with equal intensities. Both experimental and numerical results reveal that the discontinuities present in the PCS crystal play a negligible role on the $\mathrm{THz}$ beam focusing properties as the $\mathrm{THz}$ intensity distribution at the focus is not significantly influenced by the diffraction from the emitter gap.

In the focal region the positive interference between the individual electromagnetic transients results in the strong electric field shown in Fig. 2. Figure 4 illustrates the evolution of the electric field along the focal region $\left(|z|<1.5 z_{\text {Rayleigh }}\right)$, where the individual beams are spatially confined (insets Fig. 4). A clear polarity reversal of the field due to the Gouy phase shift $[18,19]$ is observed while the pulse envelope is not altered.

The corresponding Gouy phase shift (black dots) is in agreement with theoretical expectations (fit). The coherent positive interference at the focus demonstrated here is fundamental to reach the highest $\mathrm{THz}$ field strength and proofs the power of the PCS approach for up-scaling the $\mathrm{THz}$ pulse energy while keeping the excellent spatiotemporal beam properties in the focus.

Single organic DSTMS crystal offers conversion efficiency around $1 \%$ [7] and a similar value is expected for

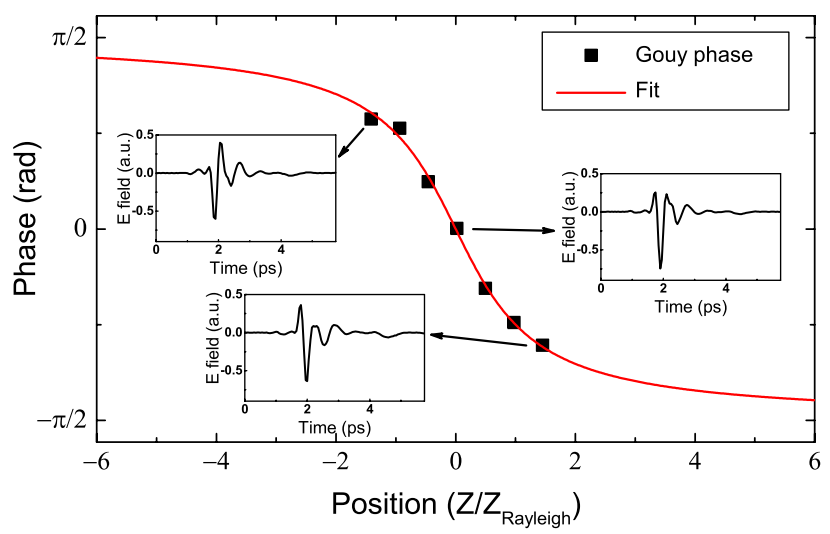

FIG. 4 (color online). Measured Gouy phase shift (dots) and polarity reversal of the net electric field (insets) across the focus, measured by electro-optical sampling.

PCS. Figure 5 illustrates the THz pulse energy (per unit of emitting surface) and the conversion efficiency of PCS as a function of the pump fluence. The $\mathrm{THz}$ pulse energy is linearly increasing for larger pump fluence [Fig. 5(a)] and reaches a peak energy density of $170 \mu \mathrm{J} / \mathrm{cm}^{2}$ at $18 \mathrm{~mJ} / \mathrm{cm}^{2}$, which is close to the damage threshold of the crystal $\left(>20 \mathrm{~mJ} / \mathrm{cm}^{2}\right)$. The pump-to-THz efficiency [Fig. 5(b)] shows a similar linear increase up to $1.1 \%$ at $7 \mathrm{~mJ} / \mathrm{cm}^{2}$ pump, before saturation effects take place. For the PCS crystal (black squares) the THz yield is slightly
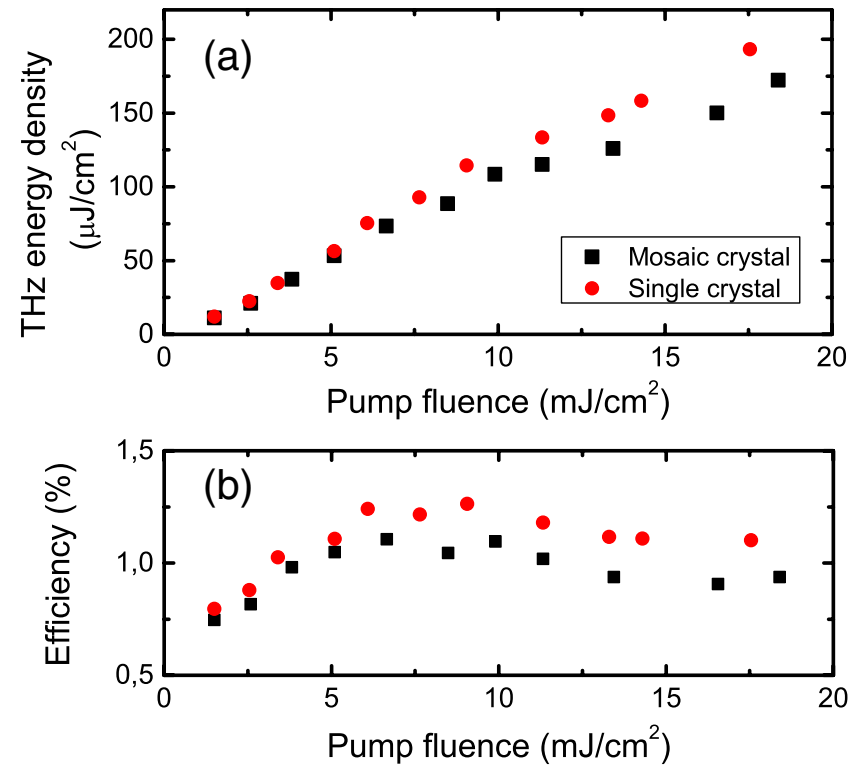

FIG. 5 (color online). (a) THz pulse energy density for PCS DSTMS nonlinear crystal (black dots). The THz energy density linearly increases for larger pump fluence, up to the damage threshold of the crystal $\left(>20 \mathrm{~mJ} / \mathrm{cm}^{2}\right)$. The characteristics are similar to the small-size single crystal (red circles). (b) Corresponding conversion efficiency in dependence of the pump fluence. The $\mathrm{THz}$ pulse energy has been recorded by means of a calibrated Golay cell. 


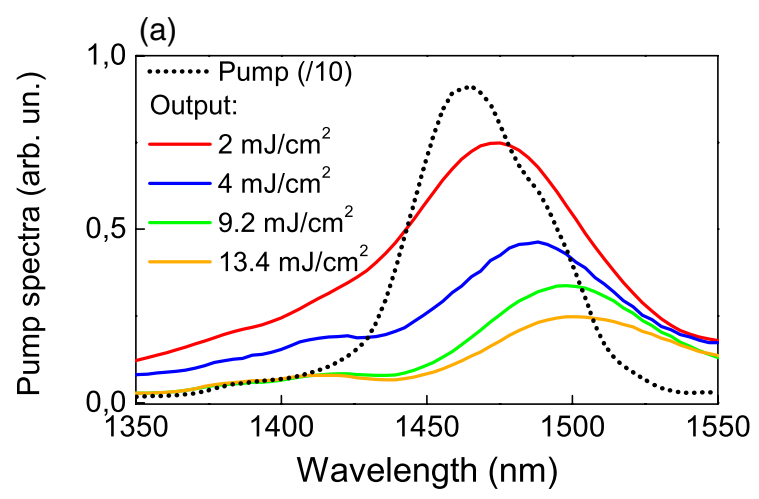

(b)

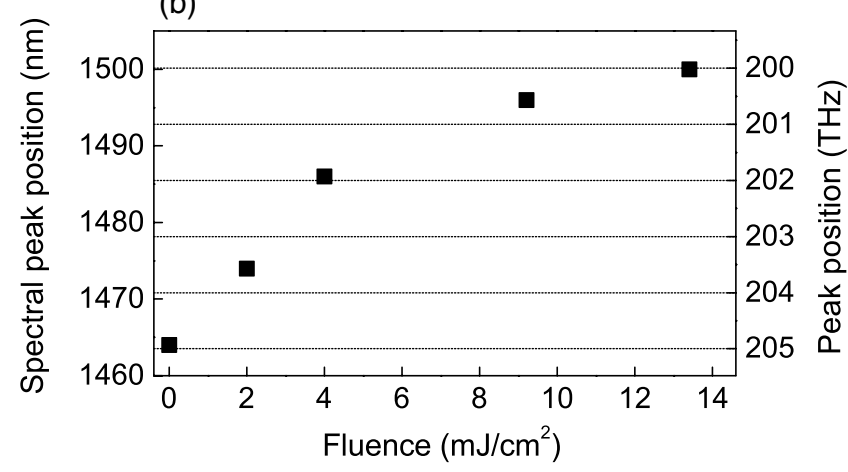

FIG. 6 (color online). (a) Pump spectra and (b) corresponding spectral peak redshift caused by cascaded optical rectification in the organic crystal. The cascading OR order and the efficiency in the $\mathrm{THz}$ generation are governed by the pump fluence.

lower (on average 15\% less) with respect to the single, small-size crystal (red circles) due to the loss and scattering occurring at the gaps and the loss of pump power at the substrate interfaces. Using the full area of the PCS crystal with the maximum pump fluence would result in $\approx 0.68 \mathrm{~mJ}$ energy per $\mathrm{THz}$ pulse.

The energy conversion efficiency is even higher if we consider that almost $70 \%$ of input pump energy is absorbed during propagation through the crystal and, on average, only $55 \%$ of the OPA energy contributes effectively to $\mathrm{THz}$ generation. The observed high efficiency is due to cascaded OR. During this process a pump photon emitting one $\mathrm{THz}$ photon undergoes a small redshift and then contributes again to $\mathrm{THz}$ generation by $\mathrm{OR}$, according to the relation $h v_{p}^{n}=h v_{p}^{n-1}-h v_{\mathrm{THz}}$. Due to this cascaded $\chi^{(2)}$ nonlinearity the initial pump spectrum undergoes a series of shifts toward longer wavelength due to emission of multiple THz photons. This effect depends strongly on the pump fluence, which is illustrated in Fig. 6(a). The pump peak wavelength shifts by more than $40 \mathrm{~nm}$ from $1460 \mathrm{~nm}(205 \mathrm{THz})$ to $1500 \mathrm{~nm}$ (200 THz) for an increase in pump fluence [Fig. 6(b)]. Similar, but less pronounced redshifting have been reported for other OR crystals [20]. In our experiment cascaded OR leads to a much higher $\mathrm{THz}$ conversion efficiency than theoretical predicted by the Manley-Rowe limit $N\left(h v_{\mathrm{THz}}\right) \leq N\left(h v_{p}\right)$. We calculated $N\left(h v_{\mathrm{THz}}\right) / N\left(h v_{p}\right) \approx 1.4$, taking into account

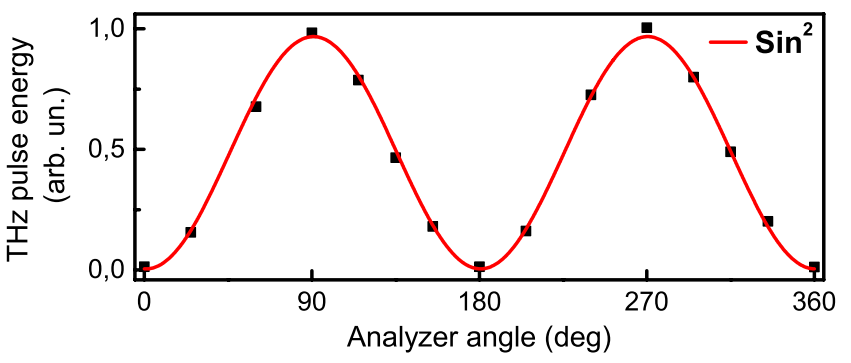

FIG. 7 (color online). Energy transmission measurements as function of analyzer angle. The measurement, fitted to a $\sin ^{2}$, demonstrates excellent linear polarization.

the pump absorption and the spectral center frequency of $2.9 \mathrm{THz}$. We note that the recycling of photons contributing to sequential OR processes is similar to the principle of quantum cascade lasers. There the same electron contributes several times to $\mathrm{THz}$ emission by passing through subsequent quantum wells [21].

To complete the $\mathrm{THz}$ beam characterization we investigated the beam polarization purity. The polarization was determined by recording the $\mathrm{THz}$ energy after a polarizer (analyzer) positioned at different angles. The measured power as function of analyzer angle is shown in Fig. 7 and is in excellent agreement with a squared sinusoidal fit as expected from a linearly polarized beam.

In conclusion we have proven that the concept of a large, partitioned $\mathrm{THz}$ emitter surface is suited for up-scaling the $\mathrm{THz}$ field strength and energy of compact, laser-driven tabletop systems. Despite the presence of discontinuities in the PCS a focus spot size close to its diffraction limit could be achieved. The DSTMS-based PCS provides a high laserto- $\mathrm{THz}$ conversion efficiency of $\approx 1 \%$, due to cascaded optical rectification, which results in a maximum emitted $\mathrm{THz}$ energy density of $170 \mu \mathrm{J} / \mathrm{cm}^{2}$. The coherent positive interference of individual field bursts in the focus results in a single-cycle, linearly polarized $\mathrm{THz}$ transient with $0.6 \mathrm{GV} / \mathrm{m}$ electric and $2 \mathrm{~T}$ magnetic field strength in the previously hardly accessible frequency range of $1-8 \mathrm{THz}$. Further up-scaling in field is only limited by the available pump laser energy since the organic crystal structure can easily be expanded in size. Exploiting the full PCS crystal area with $20 \mathrm{~mJ} / \mathrm{cm}^{2}$ laser fluence results in singlecycle transients of up to $680 \mu \mathrm{J} \mathrm{THz}$ pulse energy and $3.6 \mathrm{GV} / \mathrm{m}(12 \mathrm{~T})$ field strength, corresponding to $2.8 \mathrm{GW}$ peak power and $3 \mathrm{TW} / \mathrm{cm}^{2}$ peak intensity. This opens an avenue toward novel nonlinear $\mathrm{THz}$ applications beyond state of the art [22-25].

We acknowledge fruitful discussion with M. Jazbinsek and C. Medrano. This work was supported by SNSF (Grant No. 51NF40-144615) in the framework of NCCR-MUST and by SwissFEL. B. M. acknowledges support from Sciex-NMS (Grant No. 12.159) and C.P. H. by SNF (Grant No. PP00P2_128493). 
[1] T. Kampfrath, K. Tanaka, and K. A. Nelson, Nat. Photonics 7, 680 (2013).

[2] M. Clerici et al., Phys. Rev. Lett. 110, 253901 (2013).

[3] H. Hirori, A. Doi, F. Blanchard, and K. Tanaka, Appl. Phys. Lett. 98, 091106 (2011).

[4] K. L. Yeh, M. C. Hoffmann, J. Hebling, and K. A. Nelson, Appl. Phys. Lett. 90, 171121 (2007).

[5] A. Schneider, M. Neis, M. Stillhart, B. Ruiz, R. U. A. Khan, and P. Günter, J. Opt. Soc. Am. B 23, 1822 (2006).

[6] C. P. Hauri, C. Ruchert, C. Vicario, and F. Ardana, Appl. Phys. Lett. 99, 161116 (2011).

[7] C. Ruchert, C. Vicario, and C. P. Hauri, Phys. Rev. Lett. 110, 123902 (2013).

[8] C. Vicario, C. Ruchert, and C. P. Hauri, J. Mod. Opt. 800242 (2013).

[9] C. Ruchert, C. Vicario, and C. P. Hauri, Opt. Lett. 37, 899 (2012).

[10] S-W. Huang, E. Granados, W.R. Huang, K.-H. Hong, L. E. Zapata, and F. X. Kärtner, Opt. Lett. 38, 796 (2013); C. Vicario, B. Monoszlai, Cs. Lombosi, A. Mareczko, A. Courjaud, J. A. Fülöp, and C.P. Hauri, Opt. Lett. 38, 5373 (2013).

[11] W. R. Huang, S.-W. Huang, E. Granados, K. Ravi, K.-H. Hong, L. E. Zapata, and F. X. Kärtner, J. Mod. Opt. 10 868547 (2013).

[12] A. Riefer, S. Sanna, A. Schindlmayr, W. G. Schmidt, Phys. Rev. B 87, 195208 (2013).
[13] J. H. Jeong et al., Sci. Rep. 3, 3200 (2013).

[14] T. J. Carrig, G. Rodriguez, T. S. Clement, A. J. Taylor, and K. R. Stewart, Appl. Phys. Lett. 66, 10 (1995).

[15] A. Gopal et al., Phys. Rev. Lett. 111, 074802 (2013).

[16] D. Daranciang, J. Goodfellow, M. Fuchs, H. Wen, S. Ghimire, D. A. Reis, H. Loos, A. S. Fisher, and A. M. Lindenberg, Appl. Phys. Lett. 99,141117 (2011).

[17] A. Trisorio, P. M. Paul, F. Ple, C. Ruchert, C. Vicario, and C. P. Hauri, Opt. Express 19, 20128 (2011).

[18] S. Feng, H. G. Winful, and R. W. Hellwarth, Opt. Lett. 23, 385 (1998).

[19] A. B. Ruffin, J. V. Rudd, J. F. Whitaker, S. Feng, and H. G. Winful, Phys. Rev. Lett. 83, 3410 (1999).

[20] K. L. Vodopyanov, Opt. Express 14, 2263 (2006).

[21] B. S. Williams, Nat. Photonics 1, 517 (2007).

[22] C. Vicario, C. Ruchert, F. Ardana-Lamas, P. M. Derlet, B. Tudu, J. Luning, and C. P. Hauri, Nat. Photonics 7, 720 (2013).

[23] A. Dienst, M. C. Hoffmann, D. Fausti, J. C. Petersen, S. Pyon, T. Takayama, H. Takagi, and A. Cavalleri, Nat. Photonics 5, 485 (2011).

[24] T. Kampfrath, A. Sell, G. Klatt, A. Pashkin, S. Mährlein, T. Dekorsy, M. Wolf, M. Fiebig, A. Leitenstorfer, and R. Huber, Nat. Photonics 5, 31 (2011).

[25] B. D. Patterson et al., Chimia 65, 323 (2011). 